\title{
Pet-keeping and its impact on asthma and allergies among preschool children in Shanghai, China
}

\author{
HUANG Chen ${ }^{1 *}$, HU Yu ${ }^{1}$, LIU Wei ${ }^{1}$, ZOU ZhiJun $^{1} \&$ SUNDELL Jan ${ }^{2}$ \\ ${ }^{1}$ School of Environment and Architecture, University of Shanghai for Science and Technology, Shanghai 200093, China; \\ ${ }^{2}$ Department of Building Science, Tsinghua University, Beijing 100084, China
}

Received October 8, 2012; accepted January 14, 2013; published online April 2, 2013

\begin{abstract}
Whether early pet-keeping is a risk factor for children's asthma and allergies remains controversial. To investigate associations between asthma, allergies and airway symptoms among children and the indoor environment, a cross-sectional study was conducted in 5 districts of Shanghai. A number of 13335 questionnaires (response rate: 85.3\%) of 4-6 year old children were analyzed. Families in urban areas have more pets except for dogs than families in suburbs. Fish are the most common pets in urban districts. The prevalence of doctor-diagnosed asthma was $10.3 \%$, wheeze (ever) $28.3 \%$, rhinitis (ever) $54.1 \%$, doctor-diagnosed hay fever $12.6 \%$ and eczema (ever) $22.9 \%$. In logistic regression analyses, early furred pet-keeping was positively associated with most of the symptoms and significantly with rhinitis (ever, adjusted $\mathrm{OR}=1.41,95 \% \mathrm{CI}=1.14-1.76$ ) and doctor-diagnosed hay fever $(1.38,1.02-1.88)$. Current furred pet-keeping was significantly negatively associated with doctor-diagnosed asthma (0.57, 0.39-0.83). Persistent furred pet-keeping was significantly positively associated with rhinitis on pet or pollen exposure. However, current pet-keeping is not randomly distributed in the population. Children in families with "allergy" or with "pet avoidance behavior" (due to allergies in the family) have more symptoms, but have avoided cats and dogs, leading to the conclusion that such animals are "protective", namely the "Healthy Pet-Keeping" effect. Moreover, rodents and birds are risks for children's health. Fish-keeping is also seemingly a risk. This study indicates that early pet-keeping is a risk factor for asthma and allergies in families with a history of allergies, and part of residents in Shanghai have pet-avoidance behavior.
\end{abstract}

pet, asthma, allergies, children, avoidance behavior

Citation: Huang C, Hu Y, Liu W, et al. Pet-keeping and its impact on asthma and allergies among preschool children in Shanghai, China. Chin Sci Bull, 2013, 58: 4203-4210, doi: 10.1007/s11434-013-5679-4

It has long been discussed whether pet-keeping in early childhood is a risk or protective factor for allergies among children. The relevant studies are partly summarized by some reviews [1-4] and a meta-analysis [5]. The effect is still uncertain. Although pet-keeping might increase sensitization, it could alleviate the degree of the symptoms-development [1] and prevent children from getting hay fever [6]. Some studies have shown that there were no significant associations between pet-keeping and allergies in childhood [7-9] or a "protective" effect on early pet-keeping [4,10-16]. But some other studies revealed that the "protective" effect might be confounded by parental avoidance behavior or

*Corresponding author (email: huangc@usst.edu.cn, hcyhyywj@163.com) selection of particular pet species [10,16,17]. Still other studies have had different findings and concluded that petkeeping in the home is a risk factor for allergies [2,3,17-22]. Risk factors which are not randomly distributed in the study population can be confounded by bias. Family avoidance behavior of pet-keeping ("avoiding having pet" or "got rid of pet" due to allergies in the family) is one such possible confounder [23]. In Sweden [17] and Bulgaria [18], children in "avoidance" families were found to have more asthma and allergy, leading to the inference that pet-keeping may is "protective" in cross-sectional analyses. In Sweden [17], there had been a large campaign prior to the study to educate the general population about asthma, allergies and risk factors (The Allergy Year 1995). Thus, pet-keeping appears 
to be "protective" in countries with a high awareness of petkeeping risk, but a "risk" in countries with less awareness. Moreover, the associations between pet-keeping and asthma and allergies differed with different economic status [22] or in different races [24].

However, most studies relating children's health to petkeeping have been undertaken in developed countries or regions. There have been few in China [8,19,25-28]. The purpose of this paper is to quantify pet-keeping in Shanghai, study the associations between pet-keeping, pet avoidance behavior and asthma and allergies among preschool children, and to analyze the effect of "pet avoidance behavior" on these associations.

\section{Methods}

\subsection{The survey}

This study is part of the China, Children, Homes, Health $(\mathrm{CCHH})$ study which is being conducted throughout China. The CCHH study focuses on associations between asthma, allergies and airway symptoms among children and exposures in the home environment (e.g. pet-keeping, building dampness, building materials and environmental tobacco smoke). The study has two phases, a cross-sectional questionnaire study, followed by a nested case-control study. This is the report on the questionnaire study in Shanghai conducted in 5 of 18 districts from April 2011 to April 2012. Parents of 17898 children from 72 randomly selected kindergartens in those districts were surveyed. Questionnaires were distributed in two ways: (1) delivered to parents in kindergartens and recovered on-site by our team members;
(2) posted to kindergartens where teachers delivered them to parents, then recovered by teachers and mailed them back to us. The survey was supported by the Shanghai Municipal Bureau of Education.

\subsection{The questionnaire and statistical analysis}

Questions about asthma and allergies were the same as in the International Study of Asthma and Allergies in Childhood (ISAAC) [29] and are shown in Table 1. Doctor-diagnosed asthma and doctor-diagnosed hay fever had been diagnosed by doctors previously and reported by parents, while all other symptoms were self-reported by parents. Questions on the residence and environment were essentially the same as in the parallel studies in Sweden [30], Bulgaria [31] and Singapore [32], but slightly modified to adapt to the characteristics of Shanghai. For pet-keeping behavior, it was asked whether the family had pets currently and/or at the time of the child's birth, and if so, what kind of pets (dog, cat, rodent, bird, fish and others). Then, parents were asked about "avoidance behavior", specifically, to state whether they had gotten rid of pets because of asthma or allergy in the family (yes/no) and whether they had avoided getting pets for the same reason (yes/no). If they answered "yes" on either one of such questions, they were considered to have pet avoidance behavior.

SPSS (version 17.0, SPSS Ltd., Chicago, Illinois, USA) was used for inputting and analyzing the data. All particular analyses were restricted in the individuals with all complete data on the all variables. The Pearson's $C h i$-squared Test $\left(\chi^{2}\right)$ was used to assess whether prevalences in exposure groups had statistically significant difference. Bivariate and multivariate

Table 1 Questions about asthma and allergies which were analyzed in this study and the prevalence among 4-6 years old children in Shanghai $(n=13335)^{\text {a) }}$

\begin{tabular}{|c|c|c|c|c|c|}
\hline \multicolumn{2}{|r|}{ Questionnaire } & \multicolumn{4}{|c|}{ Prevalence $(\%)$} \\
\hline \multirow[b]{2}{*}{ Health indicators } & \multirow[b]{2}{*}{ Questions } & \multirow[b]{2}{*}{ Total } & \multicolumn{3}{|c|}{ Pet avoidance behavior } \\
\hline & & & $\begin{array}{c}\text { Yes } \\
(n=1844)\end{array}$ & $\begin{array}{c}\text { No } \\
(n=3824)\end{array}$ & $\begin{array}{l}\text { Missing } \\
(n=7672)\end{array}$ \\
\hline Doctor-diagnosed asthma & Has your child been diagnosed with asthma by a doctor? & 10.3 & 19.0 & 7.8 & 9.5 \\
\hline Wheeze ever & $\begin{array}{l}\text { Has your child ever had wheezing or whistling in the chest at any time in the } \\
\text { past? }\end{array}$ & 28.3 & 39.1 & 24.9 & 27.5 \\
\hline Wheeze in the last 12 months & Last 12 months, has your child had wheezing or whistling in the chest? & 21.7 & 29.7 & 19.2 & 21.0 \\
\hline Cough during nights & $\begin{array}{l}\text { Last } 12 \text { months, has your child had a dry cough at night for more than two } \\
\text { weeks? }\end{array}$ & 19.5 & 26.1 & 17.5 & 18.9 \\
\hline Rhinitis ever & $\begin{array}{l}\text { Has your child ever had sneeze, or a runny, or a blocked nose without a cold } \\
\text { or flu? }\end{array}$ & 54.1 & 66.7 & 50.0 & 53.2 \\
\hline Rhinitis in the last 12 months & $\begin{array}{l}\text { Last } 12 \text { months, has your child had a problem with sneezing, or a runny, or a } \\
\text { blocked nose without a cold or flu? }\end{array}$ & 42.7 & 55.8 & 38.6 & 41.5 \\
\hline Rhinitis on pet exposure & $\begin{array}{l}\text { Last } 12 \text { months, has your child had a problem with sneezing, or a runny, or a } \\
\text { blocked nose or itchy-watery eyes after being in contact with furry animals? }\end{array}$ & 4.6 & 10.5 & 3.5 & 3.6 \\
\hline Doctor-diagnosed hay fever & Has your child been diagnosed with hay fever or allergic rhinitis by a doctor? & 12.6 & 24.1 & 9.2 & 11.5 \\
\hline Eczema ever & Has your child ever had an itchy rash last 6 months at any time in the past? & 22.9 & 33.8 & 18.8 & 22.4 \\
\hline Eczema in the last 12 months & Last 12 months, has your child had eczema at any time? & 13.2 & 21.1 & 10.8 & 12.5 \\
\hline
\end{tabular}

a) Families that avoid keeping pet or have gotten rid of pets because of allergies in the family, and all of the differences between the prevalence in the families with pet avoidance behavior and the prevalence in the families without such behavior are significant in Pearson's $C h i$-squared Test $\left(\chi^{2}\right)$ and $P \leqslant 0.001$. 
logistic regression analyses were applied. Crude odds ratio (crude OR) and adjusted odds ratio (adjusted OR) with 95\% confidence interval $(\mathrm{CI})$ were used to indicate associations. A $P \leqslant 0.05$ indicated significance in all of the statistical analyses.

\section{Results}

Totally, 15266 out of 17898 questionnaires were returned, yielding a response rate (RR) of $85.3 \%$. Children's ages ranged from 1 to 8 years old. Boys accounted for $50.9 \%$ $(n=7733)$. Questionnaires returned from urban and suburban areas were $8530(\mathrm{RR}=83.7 \%)$ and $6736(\mathrm{RR}=87.4 \%)$, respectively. The questionnaires from $4-6$ year old children $(n=$ $13335,87.4 \%$ of the total participants) were selected for analyses, including " 4 years old" $(n=5561,41.7 \%)$, "5 years old" $(n=4399,33.0 \%)$ and "6 years old" $(n=3375,25.3 \%)$. The detailed distribution of samples is shown in Figure 1.

The prevalences of asthma and allergies and the petkeeping behavior in 4-6 year old children in Shanghai are given in Tables 1 and 2. In total, $32.5 \%$ of families showed avoidance behavior. Children whose families have avoidance behavior in pet-keeping have higher prevalences of all health indicators (Table 1). Furthermore, prevalences of these symptoms were analyzed in different situations of pet-keeping and avoidance behavior (Table 2). Children exposed to pets in their homes have higher prevalences than those who have never had pets in their homes. For example, the prevalences of wheeze (ever) in "persistent pet-keeping", "current pet-keeping" and "early pet-keeping" are 29.9\%, $31.7 \%$ and $34.8 \%$ respectively, while "Never keeping a pet (reference)" is $27.1 \%$. The largest differences in prevalences between pet-keeping and pet avoidance are in "early petkeeping". We also found (data not shown in the Table 2) that urban families are more pet avoiding than suburban

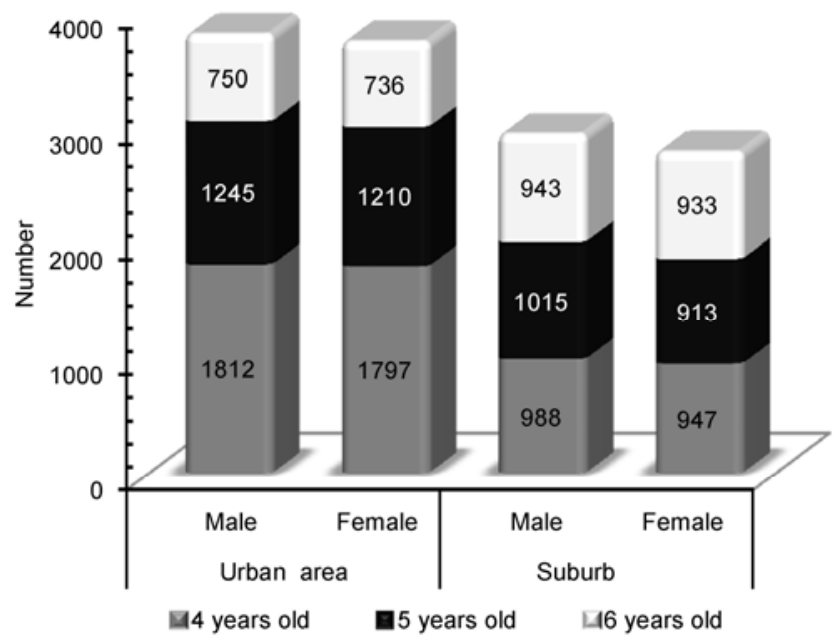

Figure 1 The distribution of children's number in different districts, ages and gender. families (totally, $42.9 \%$ vs. $19.3 \%$ ), and families with allergies in the family have more pet avoidance behavior than those without (totally, $54.8 \%$ vs. $24.5 \%$ ).

Pet species and quantities of pet-keeping are shown in Table 3. The pet-keeping ratio greatly increased from child's birth $(5.7 \%)$ to present $(14.4 \%)$. Urban families raise or raised more pets than suburban families and they were most likely to keep fish $(9.8 \%)$. Families with allergies in the family had more pets than those without, and the most common current pet in these families was fish (12.4\%). There were still pets in families with avoidance behavior, mostly fish (15.0\%). Persistently, 9.4\% of families kept pets and $37.2 \%$ of those pets were fish in total, but the most common pets were dogs in suburbs (data for Persistent petkeeping both at birth and current are not shown in the Table $3)$. Currently, fish-keeping accounts for the largest petkeeping proportion (8.9\%), followed by dog-keeping (2.4\%). However, for early pet-keeping, the most common pets were dogs (total, 3.2\%) regardless of whether families had allergies in the family $(3.5 \%)$ or not $(3.0 \%)$. Pet-keeping in Shanghai is predominantly of dogs, cats and fish. There were few other kinds of pets.

For better quantifying the specific risk of pets for children's health, integrated pet-keeping was distributed into furred pet-keeping (cat, dog and rodent or bird) and fishkeeping and analyzed for their associations with relative symptoms (Tables 4 and 5). Bivariate logistic regression analysis (Crude OR) showed that current furred pet-keeping had negatively significant (OR, 95\% CI $<1, P \leqslant 0.05)$ associations with doctor-diagnosed asthma and hay fever (Table 4), while early furred pet-keeping was positively ( $\mathrm{OR}>1)$ associated with all of the symptoms except for rhinitis on pet or pollen exposure, and significantly $(P \leqslant 0.05)$ so with wheeze, cough, rhinitis (ever or in the last 12 months), and doctor-diagnosed hay fever. Persistent furred pet-keeping was also positively associated with all symptoms except for rhinitis (ever or in the last 12 months) and eczema (ever or in the last 12 months), and significantly so with wheeze (in the last 12 months), rhinitis on pet exposure and rhinitis on pollen exposure. After adjusting for the confounding factors by multivariate logistic regression analysis (adjusted OR), significantly positive associations between certain furred pet-keeping situations and wheeze (ever or in the last 12 months), cough and eczema in the last 12 months disappeared, but still with a marginal significance.

Moreover, either persistent or current fish-keeping was significantly positively associated with almost all symptoms (Table 5). Early fish-keeping was also positively associated with all symptoms and significantly so with wheeze (ever) and cough. After adjusting for the confounding factors, most of these significant positive associations between persistent fish-keeping and symptoms disappeared, but most of such associations between current fish-keeping and symptoms still existed.

Furred pet-keeping, categorized into cat-keeping, dog- 
(1)

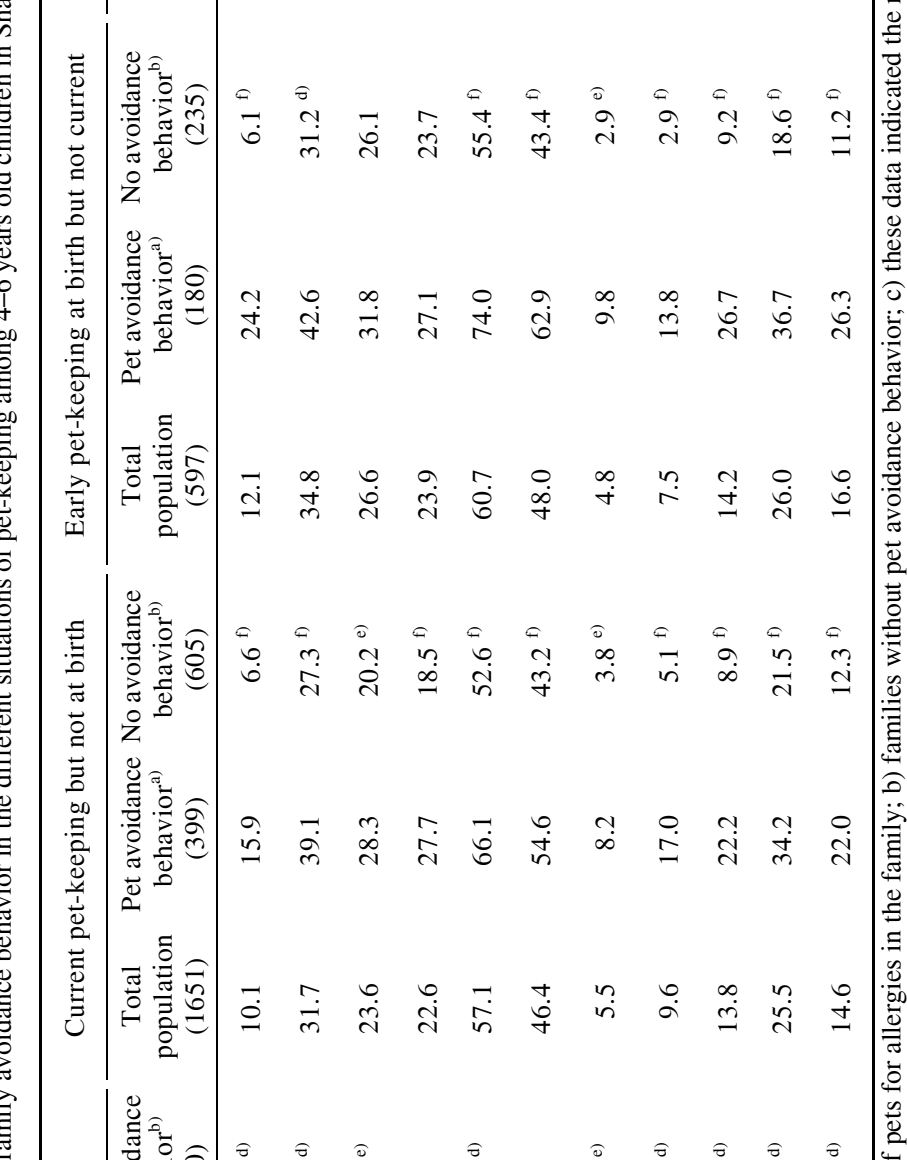

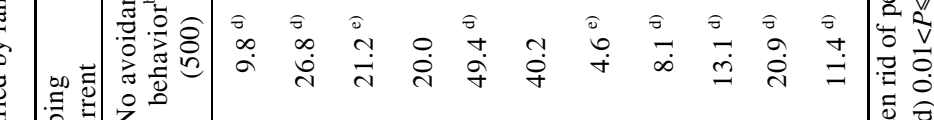

嗮

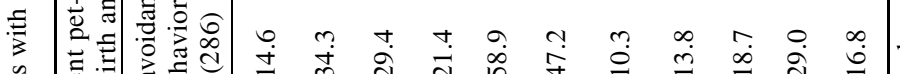

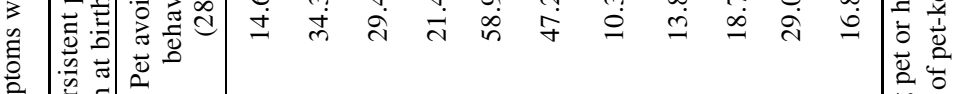

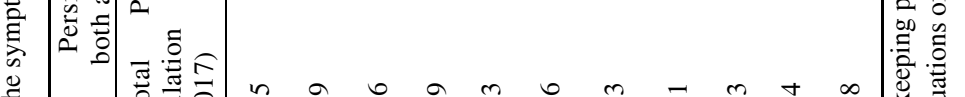

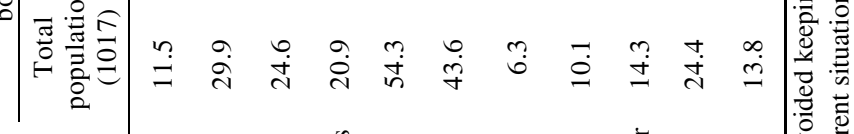

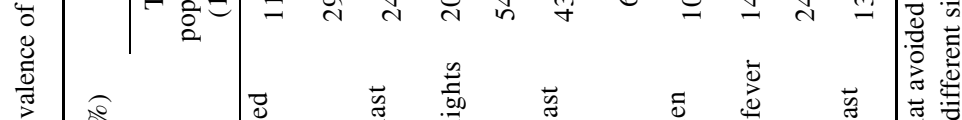

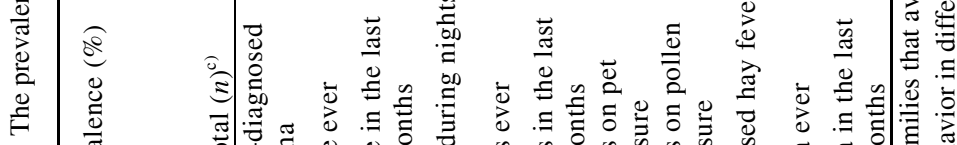

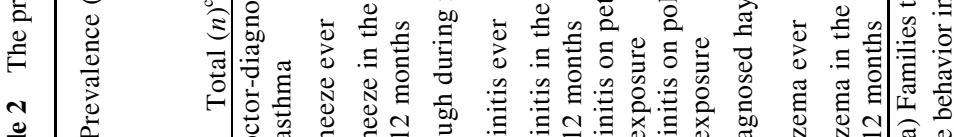

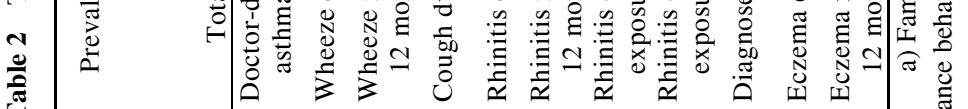

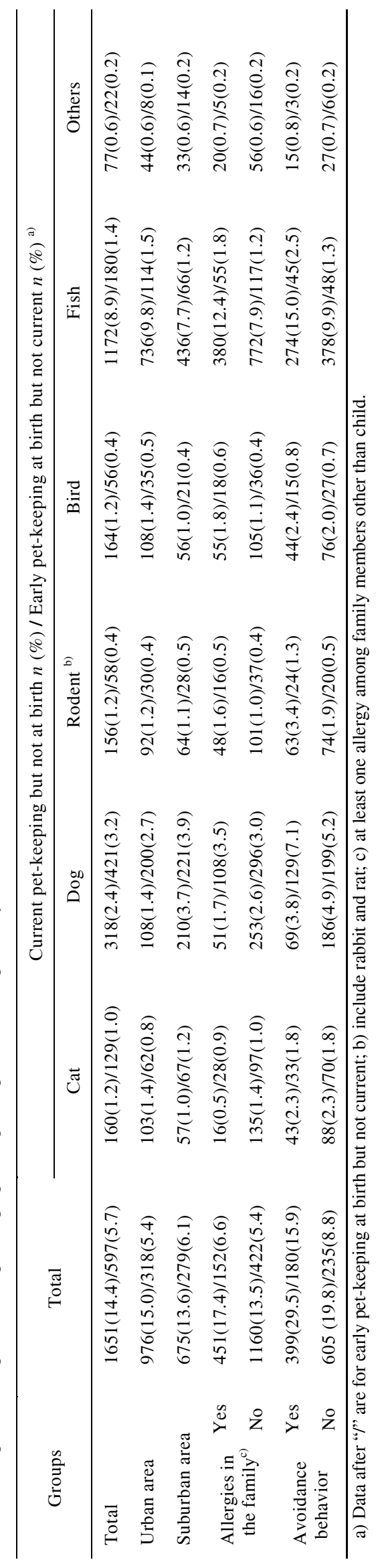



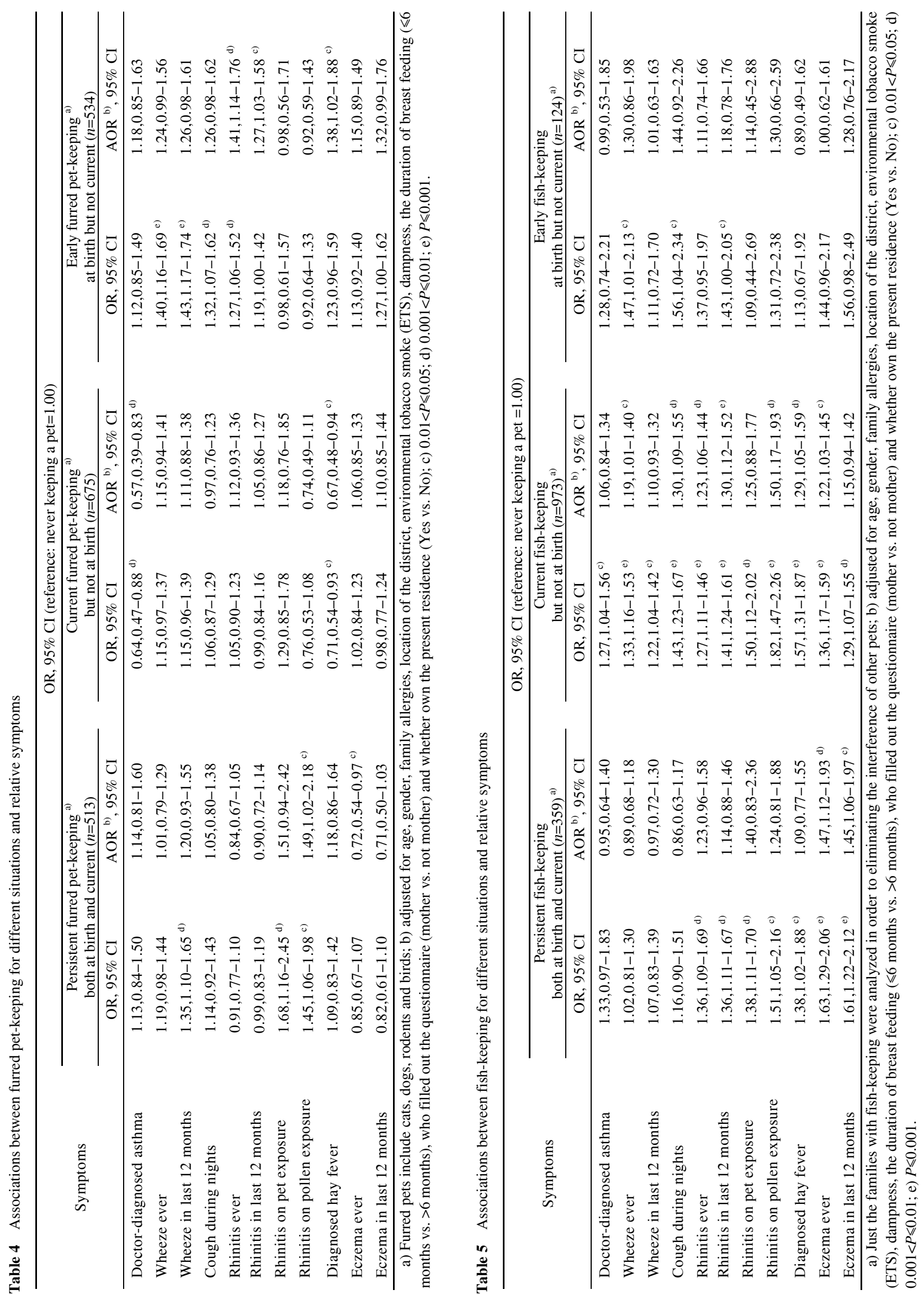
keeping and rodent-keeping or bird-keeping, was further selected to compare for associations with asthma and all allergic symptoms. Table 6 shows that early cat-keeping had positive but not significant associations with all symptoms except for rhinitis on pet (or pollen) exposure, and early dog-keeping had positive associations with all symptoms and significantly so with cough during night, rhinitis (ever), doctor-diagnosed hay fever, and eczema (in the last 12 months), while early rodent-keeping or bird-keeping had positive associations with all symptoms except for wheeze (in the last 12 months) and rhinitis on pollen exposure, and significantly so with rhinitis (ever and in the last 12 months) and doctor-diagnosed hay fever. However, these furred pets kept currently had more negative associations with related symptoms and current dog-keeping was significantly negatively associated with doctor-diagnosed asthma (adjusted OR, 95\% CI: 0.41, 0.19-0.89).

\section{Discussions}

The prevalence of allergies in Shanghai children is comparable to that in western countries [33] and is generally higher than for other Chinese cities $[8,19,25,26]$. This survey indicated that the prevalence of asthma in 4-6 years old children has risen to $10.3 \%$, much higher than the $5.92 \%$ reported for Shanghai 6-14 year old children in 2007 [34].

The present study used the same questionnaires and protocols as studies in Sweden [30], Bulgaria [31] and Singapore [32], and the $\mathrm{CCHH}$ study in other Chinese cities, and had a large sample with a high response rate. Thus, the data should well represent Shanghai urban and suburban 4-6 year old children and can be compared to other similar studies. Importantly, by directly asking about avoidance be- havior, this study was better able to determine whether petkeeping during early childhood was a risk or protective factor for later development of allergies.

Compared to Sweden [17] and Bulgaria [18], Shanghai families had fewer furred pets, but more fish. People from the suburbs preferred dogs, while the majority of urban inhabitants raised fish. This might be due to Chinese culture in keeping fish and because the great majority of urban residences are apartments in areas of high population density, making fish-keeping more convenient. More Shanghai families $(32.5 \%)$ demonstrated avoidance behaviors than that has been found In Sweden (27.3\%) [17] and Bulgaria (15.0\%) [18]. And the finding that children in families with avoidance behaviors had more asthma and allergies, agreed with those from Sweden and Bulgaria.

The results in Tables 2 and 3 also showed that there are or were pets in families that have or had avoidance behavior. This suggests that many inhabitants of Shanghai are aware that specific pets (furred) are a risk factor for their children's health problems and consequently avoid them. This is particularly true for families with allergies in the family. Furthermore, the results in Table 4 showed that current furred pet-keeping was strongly negatively associated with asthma and hay fever (doctor-diagnosed), whereas early furred pet-keeping was a positively associated risk for these symptoms. It also could be inferred that most parents avoid having furred pets when their children's asthma and hay fever were diagnosed by the doctor, who would likely advise the parents to avoid these pets. Thus, families with current pet-keeping are mainly those with children who are known to not get sick with furred pet-keeping. There are more allergies, more furred pet avoidance behavior and less furred pet-keeping in families with allergies. Thus negative associations are found, and if avoidance behavior is not accounted

Table 6 Associations between early or current cat, dog and rodent or bird-keeping and relative symptoms

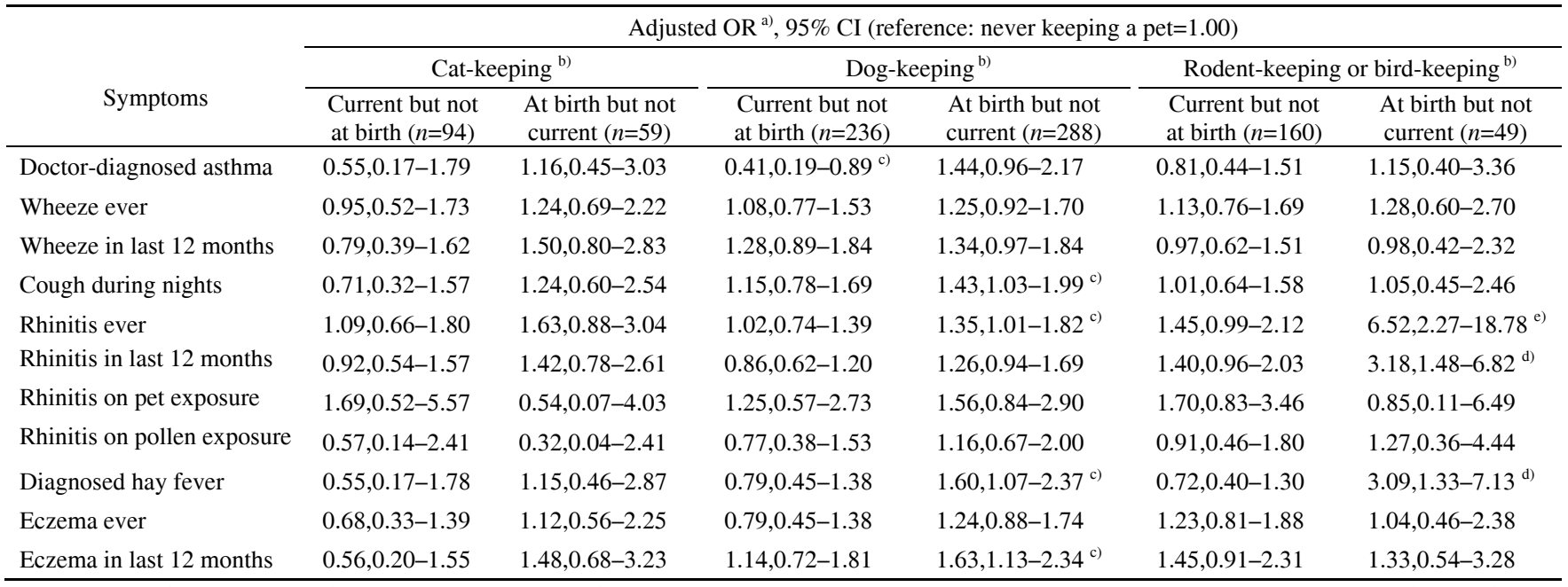

a) Adjusted for age, gender, family allergies, location of the kindergarten, environmental tobacco smoke (ETS), dampness, the duration of breast feeding ( $\leqslant 6$ months vs. $>6$ months), who filled out the questionnaire (mother vs. not mother) and whether own the present residence (Yes vs. No); b) just families kept single furred pet were analyzed in order to eliminating the interference of other furred pets; c) $0.01<P \leqslant 0.05 ; \mathrm{d}) 0.001<P \leqslant 0.01$; e) $P \leqslant 0.001$. 
for, it will confound the association between pet-keeping and children's health in a cross-sectional study, such that a "Healthy Pet-Keeping" effect would be erroneously reported $[17,18]$. Figure 2 shows our process of analysis and interpretation of results. Furthermore, when furred pet-keeping was distributed into cat-keeping, dog-keeping and rodent or bird-keeping and analyzed by the same methods, cats, dogs, rodents and birds were found to be risks for children's health, although non-significantly for cats.

Table 5 shows that fish-keeping with different situations were seemingly risk factors for children's health. Considering the data in Table 3 which suggested that families with allergic children avoided furred pets but kept fish, it is reasonable to think that fish-keeping was not the real risk factor for asthma and related symptoms in children. Although pet-keeping is increasing in families from child's birth-time till now regardless of avoidance behavior, the rates of increase in families with avoidance behavior are lower than in those without avoidance behavior, except for fish-keeping (Table 3). Thus, our data did not support keeping fish as a risk factor for children's asthma and allergic symptoms.

Above all, the results indicated that early pet-keeping was a risk factor for children's asthma and allergies, and current pet-keeping, especially cat-keeping and dog-keeping, was "protective". Rather, our data support the conclusion that the "protective" effect was confounded by "pet avoidance behavior".

\section{Conclusions}

The predominant pets in Shanghai are dogs, cats and fish. Suburban people prefer dogs, while urban people prefer fish.

The prevalences of asthma and other allergic symptoms in 4-6 year old children are greater than $10 \%$. This survey

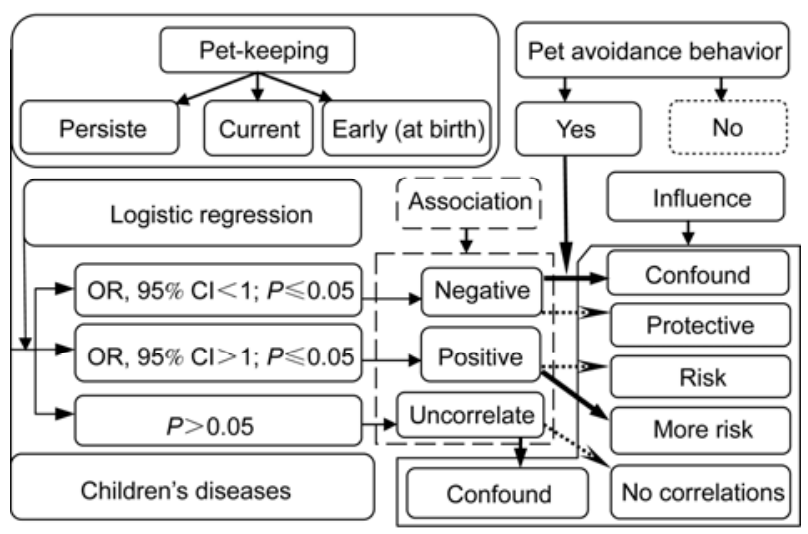

Figure 2 Flow-chart describing the process of analysis and interpretation of results. Bold arrows show how findings would be interpreted with accounting for avoidance behavior, while dashed arrows show how findings would be interpreted without accounting for avoidance behavior. Herein it was hypothesized that the uncorrelated associations (right below) were confounded by avoidance behavior, but it was not evident in our data and need to further verified. shows that early pet-keeping is a risk factor. Families' avoidance behavior, if not accounted for, can lead to the erroneous conclusion that pet-keeping is protective against asthma and related symptoms.

The authors greatly appreciate the Shanghai Municipal and District Bureau of Education for their supports. The authors thank all the parents who filled out questionnaires, the kindergartens' teachers and others who provided assistance for our survey and the teachers and students who have coordinated this survey. And a special thanks to Louise B. Weschler (Tsinghua University) for helping with the English language, to Yuexia Sun (Tianjing University) for guidance of the cross-sectional study and questionnaire, and to Zhuohui Zhao (Fudan University) for constructive comments. This work was supported by the Leading Academic Discipline Project of Shanghai Municipal Education Commission (J50502) and the National Natural Science Foundation of China (51278302 and 51108263).

1 Ahlbom A, Backman A, Bakke J, et al. "NORDPET"-A risk factor for or protection against sensitization/allergy: A Nordic interdisciplinary review of the scientific literature concerning the relationship between the exposure to pets at home, sensitization and the development of allergy. Indoor Air, 1998, 8: 219-235

2 Apelberg B J, Aoki Y, Jaakkola J J K. Systematic review: Exposure to pets and risk of asthma and asthma-like symptoms. J Allergy Clin Immunol, 2001, 107: 455-460

3 Chen C M, Tischer C, Schnappinger M, et al. The role of cats and dogs in asthma and allergy-A systematic review. Int J Hyg Environ Health, 2010, 213: 1-31

4 Lodge C J, Allen K J, Lowe A J, et al. Perinatal cat and dog exposure and the risk of asthma and allergy in the urban environment: A systematic review of longitudinal studies. Clin Dev Immunol, 2012, doi: $10.1155 / 2012 / 176484$

5 Takkouche B, González-Barcala F J, Etminan M, et al. Exposure to furry pets and the risk of asthma and allergic rhinitis: A meta-analysis. Allergy, 2008, 63: 857-864

6 Matheson M C, Dharmage S C, Abramson M J, et al. Early-life risk factors and incidence of rhinitis: Results from the European Community Respiratory Health Study-An international population-based cohort study. J Allergy Clin Immunol, 2011, 128: 816-823

7 Celedón J C, Litonjua A A, Ryan L, et al. Exposure to cat allergen, maternal history of asthma, and wheezing in first 5 years of life. Lancet, 2002, 360: 781-782

8 Solo P, Xia J, Johnson C A, et al. Indoor allergens, asthma, and asthma-related symptoms among adolescents in Wuhan, China. Ann Epidemiol, 2004, 14: 543-550

9 Lodge C J, Lowe A J, Gurrin L C, et al. Pets at birth do not increase allergic disease in at-risk children. Clin Exp Allergy, 2012, 42: 13771385

10 Nafstad P, Magnus P, Gaarder P I, et al. Exposure to pets and atopyrelated diseases in the first 4 years of life. Allergy, 2001, 56: 307-312

11 Ownby D R, Johnson C C, Peterson E L. Exposure to dogs and cats in the first year of life and risk of allergic sensitization at 6 to 7 years of age. JAMA, 2002, 288: 963-972

12 Perzanowski M S, Rönmark E, Platts-Mills T A E, et al. Effect of cat and dog ownership on sensitization and development of asthma among preteen age children. Am J Respir Crit Care Med, 2002, 166: 696-702

13 Oberle D, Mutius E, Kries R. Childhood asthma and continuous exposure to cats since the first year of life with cats allowed in the child's bedroom. Allergy, 2003, 58: 1033-1036

14 Mandhane P J, Sears M R, Poulton R, et al. Cats and dogs and the risk of atopy in childhood and adulthood. J Allergy Clin Immunol, 2009, 124: 745-750

15 Gaffin J M, Spergel J M, Boguniewicz M, et al. Effect of cat and daycare exposures on the risk of asthma in children with atopic dermatitis. Allergy Asthma Proc, 2012, 33: 282-288

16 Anyo G, Brunekreef B, Meer G, et al. Early, current and past pet 
ownership: Associations with sensitization, bronchial responsiveness and allergic symptoms in school children. Clin Exp Allergy, 2002, 32: 361-366

17 Bornehag C G, Sundell J, Hagerhed L. Pet-keeping in early childhood and airway, nose and skin symptoms later in life. Allergy, 2003, 58: 939-944

18 Naydenov K, Popov T, Mustakov T, et al. The association of pet keeping at home with symptoms in airways, nose and skin among Bulgarian children. Pediatr Allergy Immunol, 2008, 19: 702-708

19 Dong G H, Ma Y N, Ding H L, et al. Pets keeping in home, parental atopy, asthma, and asthma-related symptoms in 12910 elementary school children from northeast China. Indoor Air, 2009, 19: 166-173

20 Lombardi E, Simoni M, Grutta S L. Effects of pet exposure in the first year of life on respiratory and allergies in 7-yr-old children. The SIDRIA-2 study. Pediatr Allergy Immunol, 2010, 21: 268-276

21 Chen Y C, Tsaia C H, Leo Lee Y L. Early-life indoor environmental exposures increase the risk of childhood asthma. Int J Hyg Environ Health, 2011, 215: 19-25

22 Brunekreef B, Mutius E V, Wong G, et al. Exposure to cats and dogs, and symptoms of asthma, rhinoconjunctivitis, and eczema. Epidemiology, 2012, 23: 742-750

23 Bertelsen R J, Carlsen K C L, Granum B, et al. Do allergic families avoid keeping furry pets? Indoor Air, 2010, 20: 187-195

24 Sun Y X, Sundell J. Life style and home environment are associated with racial disparities of asthma and allergy in Northeast Texas children. Sci Total Environ, 2011, 409: 4229-4234

25 Zheng T Z, Niu S R, Lu B Y, et al. Childhood asthma in Beijing, China: A population-based case-control study. Am J Epidemiol, 2002, 156: 977-983
26 Wang S L, Guo X B, Zhang J L. Study on the effects of ambient air pollution on respiratory disease and symptoms among school-age children in Beijing (in Chinese). J Environ Health, 2004, 21: 41-44

27 Zheng Q, Guo Y. Impact of indoor environmental factors on bronchial asthma (in Chinese). Chin J Asthma, 2011, 5: 138-143

28 Dong W, Sheng J, Gu X M, et al. Allergens and their relationships to childhood bronchial asthma in the Jiading District of Shanghai (in Chinese). Chin J Contemp Pediatr, 2012, 14: 521-523

29 Asher M I, Keil U, Anderson H R, et al. International study of asthma and allergies in childhood (ISAAC): Rationale and methods. Eur Respir J, 1995, 8: 483-491

30 Engman L H. Indoor environmental factors and its associations with asthma and allergy among Swedish pre-school children. Doctoral Dissertation. Lund: Lund University, 2006

31 Naydenov K G. On the association between home exposure and asthma and allergies among children in Bulgaria/The ALLHOME Study/. Doctoral Dissertation. Lyngby: Technical University of Denmark and ICIEE, 2007

32 Zuraimi M S. Child care centre and home exposures among preschool children in Singapore and their associations with asthma, allergies and respiratory symptoms. Doctoral Dissertation. Singapore: National University of Singapore, 2008

33 Asher M I, Montefort S, Bjökstén B, et al. Worldwide time trends in the prevalence of symptoms of asthma, allergic rhinoconjunctivitis, and eczema in childhood: ISAAC Phases One and Three repeat multicountry cross-sectional surveys. Lancet, 2006, 368: 733-743

34 Yuan D, Shen C L, Jiang Z H, et al. An investigation on the prevalence of asthma and its influence factors among school age children in Shanghai (in Chinese). J Environ Occup Med, 2007, 24: 573-576

Open Access This article is distributed under the terms of the Creative Commons Attribution License which permits any use, distribution, and reproduction in any medium, provided the original author(s) and source are credited. 